\title{
Calcitonin gene-related peptide enhances osteogenic differentiation and recruitment of bone marrow mesenchymal stem cells in rats
}

\author{
SEN JIA ${ }^{1,2^{*}}$, SHI-JIAN ZHANG ${ }^{3 *}$, XU-DONG WANG ${ }^{3 *}$, ZI-HUI YANG $^{2}$, YA-NAN SUN ${ }^{4}$, \\ ANAND GUPTA ${ }^{5}$, RUI HOU ${ }^{2}$, DE-LIN LEI ${ }^{2}$, KAI-JIN HU ${ }^{2}$, WEI-MIN YE $^{3}$ and LEI WANG ${ }^{2,3}$ \\ ${ }^{1}$ Department of Oral and Maxillofacial Surgery, School of Stomatology Xi'an Medical University; \\ ${ }^{2}$ State Key Laboratory of Military Stomatology, Department of Oral and Maxillofacial Surgery, School of Stomatology, \\ The Fourth Military Medical University, Xi'an, Shaanxi 710032; ${ }^{3}$ Department of Oral and Maxillofacial-Head and Neck \\ Oncology, Ninth People's Hospital, Shanghai JiaoTong University School of Medicine, Shanghai Key Laboratory of \\ Stomatology and Shanghai Research Institute of Stomatology, National Clinical Research Center of Stomatology, \\ Shanghai 200011; ${ }^{4}$ State Key Laboratory of Military Stomatology, Department of Pediatric Stomatology, \\ School of Stomatology, The Fourth Military Medical University, Xi'an, Shaanxi 710032, P.R. China; ${ }^{5}$ Department of \\ Oral and Maxillofacial Surgery, Government Medical College and Hospital, Chandigarh, Haryana 160030, India
}

Received July 26, 2018; Accepted April 3, 2019

DOI: $10.3892 /$ etm.2019.7659

\begin{abstract}
The present study evaluated the effects of calcitonin gene-related peptide (CGRP) on bone marrow mesenchymal stem cells (BMMSCs) in vitro and in a rat model of mandibular distraction osteogenesis (MDO). Rat BMMSCs were isolated then treated with CGRP or CGRP antagonist (CGRP8-37). The proliferation and migration ability of BMMSCs was determined using 5-bromo-2'-deoxyuridine and Transwell assays, respectively. Osteogenic-related gene expression was analyzed with reverse transcription-quantitative polymerase chain reaction. For the in vivo analysis, thirty MDO rats were randomly assigned to control, CGRP or CGRP8-37 groups. To evaluate the mobilization of BMMSCs, nestin and stromal cell-derived factor 1 (SDF-1) were detected by immunohistochemistry and ELISA. Rats were sacrificed following 14 days and new bone formation was assessed by histological and micro-computed
\end{abstract}

Correspondence to: Professor Lei Wang or Dr Wei-Min Ye, Department of Oral and Maxillofacial-Head amd Neck Oncology, Ninth People's Hospital, Shanghai JiaoTong University School of Medicine, Shanghai Key Laboratory of Stomatology and Shanghai Research Institute of Stomatology, National Clinical Research Center of Stomatology, 639 Zhizaoju Road, Shanghai 200011, P.R. China

E-mail: wangleizyh@aliyun.com

E-mail: 285772228@qq.com

*Contributed equally

Key words: calcitonin gene-related peptide, distraction osteogenesis, bone marrow mesenchymal stem cells tomography analysis. In the in vitro results, the CGRP group demonstrated significantly higher migration and proliferation, as well as enhanced alkaline phosphatase and runt-related transcription factor 2 expression compared with the control. In the in vivo experiments, bone mineral density of the newly formed bone in the CGRP group was significantly higher than controls. The nestin and SDF-1 expression in the CGRP group was also significantly upregulated. In conclusion, the present study demonstrated that CGRP administration increased new bone formation, possibly via enhancing BMMSC migration and differentiation in MDO rats.

\section{Introduction}

Craniofacial defects can be caused by inflammation, trauma, tumor and congenital malformations amongst other things. For reconstruction of defects or deformities in oral and maxillofacial regions, distraction osteogenesis (DO) techniques have been broadly applied to regenerate bone tissues and are considered to be the gold-standard treatment. However, the longer period required for bone consolidation may lead to clinical complications such as fracture of the distraction device or infections (1). Therefore, acceleration of bone formation is urgently required.

The nervous system has been demonstrated to have pivotal regulatory effects on the metabolism and repair of bones $(2,3)$. Damage to peripheral nerves is often followed by osteoporosis (4). Our previous study reported that a locally applied nerve growth factor (NGF) promotes the rehabilitation of the inferior alveolar nerve (IAN) (5). In addition, delivery of human NGF $\beta$ in gels accelerates bone regeneration in DO rabbit models (6). Skeletal sensory terminals can secrete various neurotransmitters including substance $\mathrm{P}$, calcitonin gene-related peptide (CGRP), which regulates the metabolism of osteoblasts and 
osteoclasts $(7,8)$. In a bilateral periodontal defect model, the osteoprotegerin expression levels decrease along with CGRP when rats undergo transection of the left inferior alveolar nerve (9). Furthermore, the secretion of CGRP from sensory peptidergic fibers in the periosteum and bone is functional during load-induced bone regeneration (10). However, it remains unclear whether CGRP accelerates bone regeneration in DO by targeting bone marrow mesenchymal stem cells (BMMSCs). CGRP8-37 is the most widely used selective pharmacological antagonist of CGRP (11). In the present study, the effects of CGRP and CGRP-37 on BMMSCs were investigated in vitro and in vivo in a rat model of mandibular DO.

\section{Materials and methods}

Rat BMMSC culture and identification. A total of 4 male Sprague-Dawley rats (6 weeks old; 60-80 g) from the animal care center of the Fourth Military Medical University were housed under specific pathogen-free conditions $\left(22^{\circ} \mathrm{C} ; 12-\mathrm{h}\right.$ light/dark cycle; $50 \%$ humidity) with free access to food pellets and tap water. Briefly, rats were sacrificed and mandibles were resected aseptically $(12,13)$. The cell suspension was prepared by repeated aspiration through a 20 -gauge needle. The collected cells were then seeded into 6 well plates at a density of $1 \times 10^{6}$ cells per well and incubated under standard conditions $\left(5 \% \mathrm{CO}_{2}, 37^{\circ} \mathrm{C}\right)$. The culture medium consisted of $\alpha$-minimum essential medium ( $\alpha \mathrm{MEM}$ ) supplemented with $10 \%(\mathrm{v} / \mathrm{v})$ fetal bovine serum (FBS), $0.25 \mu \mathrm{g} / \mathrm{ml}$ Fungizone, and $1 \%(\mathrm{v} / \mathrm{v})$ penicillin and streptomycin (all purchased from Sigma-Aldrich; Merck KGaA).

To characterize rat BMMSCs, the morphology of adherent BMMSCs was observed using a phase contrast microscope (Nikon TE 2000-U; Nikon Corporation). Immunofluorescence staining of MSC markers thy-1 cell surface antigen (CD90) and integrin subunit $\beta 1$ (CD29) was performed using a standard procedure. Cells were fixed for $30 \mathrm{~min}$ at room temperature in $4 \%$ paraformaldehyde, washed twice with PBS then treated with $0.1 \%$ Triton $\mathrm{X}-100$ at room temperature for $1-2$ min to permeate the cell membrane. In brief, cells were blocked with $5 \%$ bovine serum albumin (BSA)/PBS $\left(1 \mathrm{~h}, 25^{\circ} \mathrm{C}\right)$, then incubated in $1 \% \mathrm{BSA} / \mathrm{PBS}$ with either isotype controls, anti-CD29 (cat. no. 610468; 1:1,000; BD Biosciences) or anti-CD90 antibodies (cat. no. 554893; 1:1,000; BD Biosciences) for $12 \mathrm{~h}$ at $4^{\circ} \mathrm{C}$. The cells were then washed three times with $0.5 \%$ BSA/PBS. Samples were then incubated in TRITC goat anti-rabbit fluorescent secondary antibody (cat. no. 150077; 1:200; Abcam). Stained cells were observed using a fluorescence microscope (magnification, x400; DXM 1200F; Nikon Corporation). In addition, the multidifferentiation potential of rat BMMSCs was determined using adipogenic or osteogenic media followed by Oil Red (Sigma-Aldrich, Merck KGaA) or Alizarin Red staining (ARS) (Sigma-Aldrich; Merck KGaA), respectively.

Mineralization assay. ARS is used to quantify calcium within the deposited mineral matrix. Following 3 weeks of culture, cells were stained with $2 \%$ Alizarin red for $10 \mathrm{~min}$. When bound to the calcium salts in cell matrix, Alizarin red was eluted with $10 \%$ cetylpyridinium chloride. The absorbance of supernatant aliquots $(\sim 150 \mu \mathrm{l})$ were measured at $540 \mathrm{~nm}$ using a spectrophotometer then standardized with the absorbance of crystal violet staining at $590 \mathrm{~nm}$.

Cell proliferation assessment. BMMSCs at passage 3 were cultured with $\alpha$-MEM containing $10^{-7} \mathrm{~mol} / \mathrm{l}$ CGRP, $\alpha$-MEM medium containing 10-7 mol/1 CGRP8-37 or $\alpha$-MEM medium only. 5-bromo-2'-deoxyuridine (BrdU; $3.1 \mu \mathrm{g} / \mathrm{ml}$ ) was added at $72 \mathrm{~h}$ post-seeding then incubated for $4 \mathrm{~h}$. After washing in PBS, cultures were fixed in ice-cold $70 \%$ ethanol for $10 \mathrm{~min}$ and denatured in $4 \mathrm{mmol} / \mathrm{l} \mathrm{HCl}$ for $20 \mathrm{~min}$. Samples were then incubated with mouse anti-BrdU primary antibody (cat. no. 5292S; 1:1,000; Cell Signaling Technology, Inc.) for $1 \mathrm{~h}$ at $37^{\circ} \mathrm{C}$ and then with HRP-conjugated second antibodies (cat. no. 7076S; 1:200; Cell Signaling Technology, Inc.) for $1 \mathrm{~h}$ at $\mathrm{RT}$. The samples were washed then stained with diamino-benzidine (DAB) and hematoxylin for nuclei staining. Cell proliferation was measured as a percentage of $\mathrm{BrdU}^{+}$cells relative to the total number of nuclei. Images were captured in 5 randomly selected fields per slide (magnification, $\mathrm{x} 200$ ). The BrdU ${ }^{+}$cells were counted manually by an experienced pathologist. All the experiments were conducted in triplicate.

Alkaline phosphatase (ALP) activity. Cultured BMMSCs were fixed in fresh $10 \%$ neutral buffered formalin at week 1 , 2 , and 3. Then ALP activity was determined by the commercial ALP activity kit. After all protocols were performed according to the manufacturer's instructions, the absorbance was measured at $410 \mathrm{~nm}$ with a spectrophotometer and the cell number was counted using crystal violet staining as previously reported $(14,15,16)$.

Animal study. A total of 30 male Sprague-Dawley rats (10 weeks old; $100-120 \mathrm{~g}$ ) from the animal care center of the Fourth Military Medical University were randomly divided into three groups: control, CGRP and CGRP8-37. The rats were housed under specific pathogen-free conditions $\left(22^{\circ} \mathrm{C}\right.$; 12-h light/dark cycle; $50 \%$ humidity) with free access to food pellets and tap water. This study was specifically approved by the Institutional Ethics Committee at the Fourth Military Medical University (approval number ky-034). All animals were cared for according to the guidelines published by the Animal Center for Medical Experiment at Fourth Military Medical University.

Surgical procedures. Animals were anaesthetized with 1\% pentobarbital sodium (intraperitoneal injection $30 \mathrm{mg} / \mathrm{kg}$ ). A vertical osteotomy was made in the right mandible between the molar region and the mandibular ramus. Surgeries were performed cautiously to avoid damage in the IAN. A custom-made distraction device was fixed along a plane perpendicular to the osteotomy cut. The device was activated following a latency period of 5 days, and the distraction was carried out at the rate of $0.2 \mathrm{~mm}$, twice per day for 10 days. During the distraction period, the CGRP group received a daily CGRP injection (Sigma-Aldrich; Merck KGaA; $10^{-8} \mathrm{mmol} / \mathrm{l}$, in normal saline, total volume of $0.2 \mathrm{ml}$ ), the CGRP8-37 group was given a daily injection of CGRP8-37 (Sigma-Aldrich; Merck KGaA; $10^{-6} \mathrm{~mol} / 1$, in normal saline, total volume of $0.2 \mathrm{ml}$ ), and the control group received normal saline (17). All 
A
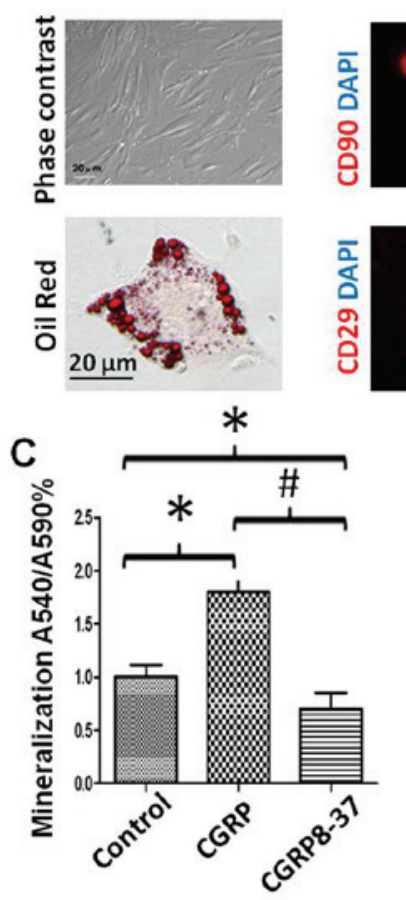
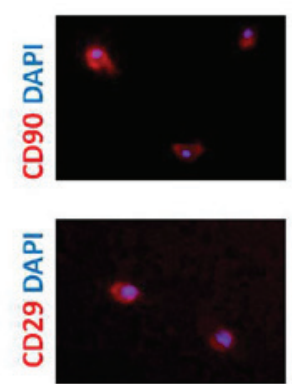

D
B

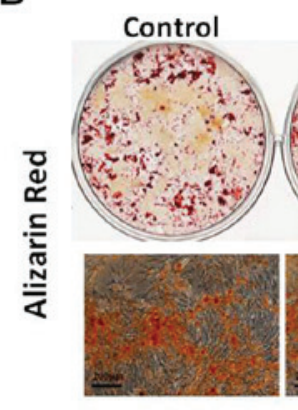

CGRP8-37

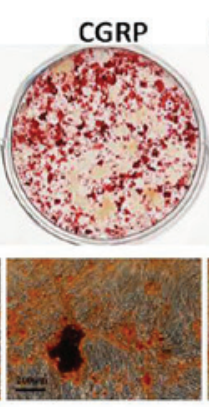

(CGRP antagonist)

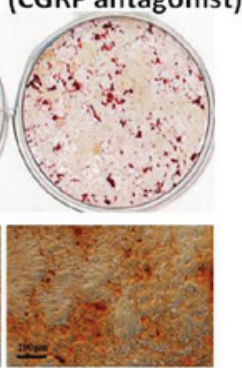

E
Control

CGRP

CGRP8-37
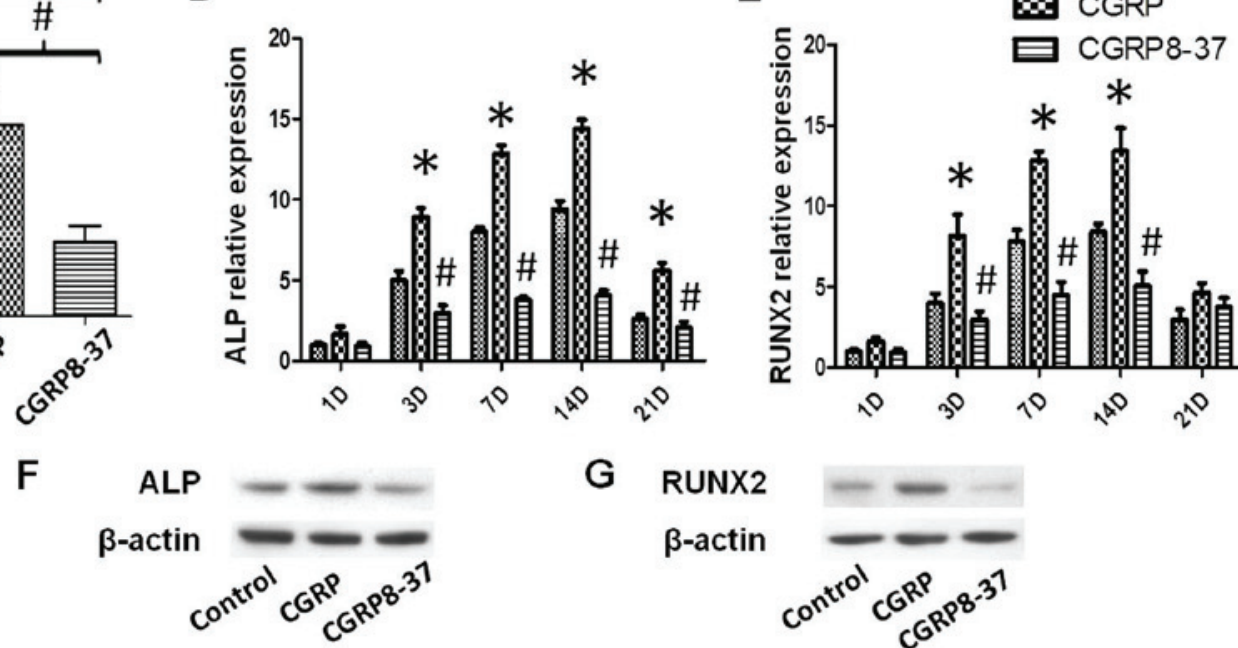

G RUNX2

$\beta$-actin

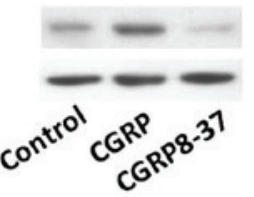

H
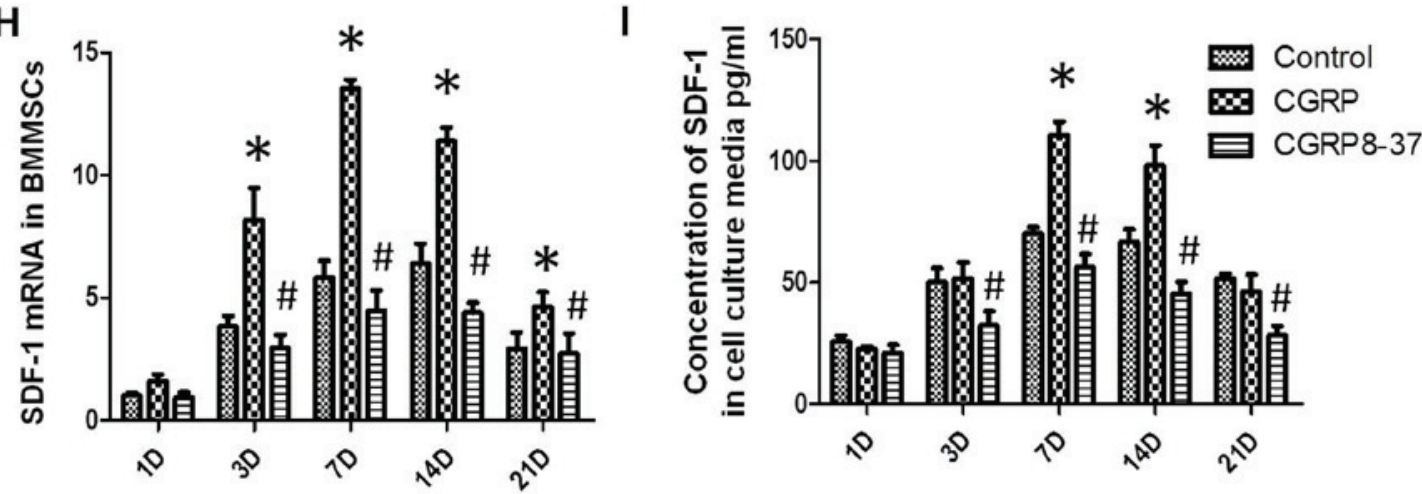

Figure 1. Effects of CGRP on osteogenic differentiation and proliferation in vitro. (A) Stem cell identification of the cultured P3 bone marrow mesenchymal stem cells extracted from the rat mandible. (B) Alizarin Red staining and (C) quantification for in vitro osteogenic differentiation. (D) mRNA expression levels of ALP and (E) RUNX2 measured by RT-qPCR. Protein expression of (F) ALP and (G) RUNX2 measured by western blot analysis. (H) Gene expression of SDF-1 measured by RT-qPCR. (I) Concentration of SDF-1 in cell culture media. ${ }^{*} \mathrm{P}<0.05$ vs. control; ${ }^{*} \mathrm{P}<0.05$ vs. CGRP. CGRP, calcitonin gene-related peptide; ALP, alkaline phosphatase; RUNX2, runt-related transcription factor 2; RT-qPCR, reverse transcription-quantitative PCR; SDF-1, stromal cell-derived factor 1; BMMSCs, bone marrow mesenchymal stem cells; CD29, integrin subunit $\beta 1$; CD90, thy-1 cell surface antigen; $D$, days.

the drugs were injected into the local callus at the distraction side percutaneously. Following 14 days of treatment, animals were sacrificed and mandibular tissues were collected for histological and micro-computed tomography (micro-CT) analysis. All the analyses were conducted blinded.

Micro-CT evaluation. All mandibular samples were examined with a Micro-CT system (Inveon CT; Siemens AG) at the standard resolution (1888x2048 pixels). Each mandibular sample included $\sim 1,000$ images with an isotropic voxel size of $15 \mu \mathrm{m}$. The scanning was set at $500 \mathrm{~mA}, 80 \mathrm{kV}$, and the integration time was $800 \mathrm{msec}$. The bone and accurate 3-dimensional data sets were isolated using the same optimal thresholds from all segmented images. The region of interest was defined as a rectangle of $2.5 \times 10 \mathrm{~mm}$ covering the distraction gap. The micro-CT measurements contained both bone mineral density (BMD) and bone volume/total volume (BV/TV), which indicates the portion of the mineralized tissue in bony defects. The newly generated BV in the distraction gap was quantified and compared to the total distraction area. Following micro-CT evaluation, all samples underwent histological analysis. 

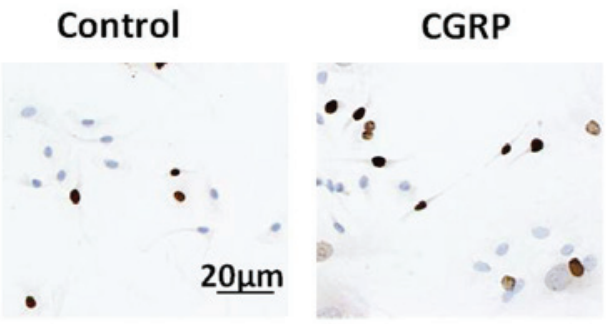

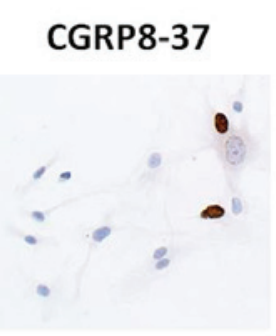

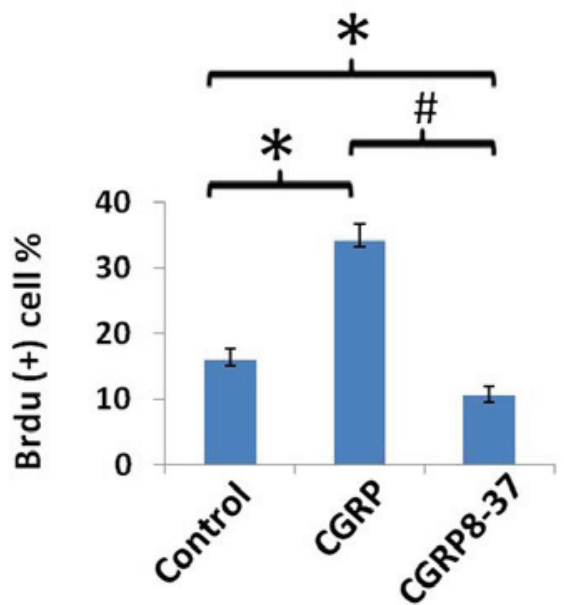

Figure 2. Effects of CGRP on osteogenic differentiation and proliferation in vitro. Micrographs demonstrating BrdU incorporation (brown staining) and hematoxylin (blue staining, nuclei). Quantification of $\mathrm{BrdU}^{+}$cells. ${ }^{*} \mathrm{P}<0.05$ vs. control; ${ }^{*} \mathrm{P}<0.05$ vs. CGRP. CGRP, calcitonin gene-related peptide; BrdU, 5-bromo-2'-deoxyuridine.

BMD analysis and immunohistochemistry. The mandibular samples were fixed in $4 \%$ paraformaldehyde for $24 \mathrm{~h}$ and decalcified in $30 \%$ buffered formic acid for 7 days at room temperature. Following dehydration and cleaning, samples were stained with hematoxylin and eosin $(\mathrm{H} \& \mathrm{E})$. Analysis of the histological sections was performed using the Image-Pro Plus analysis software (v11.0; Media Cybernetics, Inc.) by a single, unbiased examiner who was blinded in this study.

For immunohistochemistry, endogenous peroxidase activity was eradicated by $3 \%$ hydrogen peroxide for $10 \mathrm{~min}$ at room temperature. Then the tissue slices were permeabilized with $0.25 \%$ Triton X-100 in PBS for 30 min, and blocked in $5 \%$ fetal bovine serum in PBS, $2 \%$ glycine and 2\% BSA for $1 \mathrm{~h}$ at room temperature. The slices were then incubated with anti-nestin primary antibodies (cat. no. 4760S; 1:1,000; Cell Signaling Technology, Inc.) overnight at $4^{\circ} \mathrm{C}$, followed by incubation with horseradish peroxidase-conjugated secondary antibodies (1:200; cat. no. 150077; Abcam) for $1 \mathrm{~h}$ at room temperature. Samples were washed and then stained with diamino-benzidine (DAB). To quantify the proportions of nestin $^{+}$cells in bone generating areas, these areas, consisting of woven bones and fibrosis, were characterized by presence of bone trabeculae and osteoid rimmed osteoblasts within a bridging callus. Nestin ${ }^{+}$cells were manually counted under a fluorescent microscope (DMLRB; Leica Microsystems, Inc.) in 5 random high-power fields (magnification, $\mathrm{x} 400$ ) per slide. Each assay was performed in triplicate.

ELISA. Rat BMMSC supernatant was analyzed for stromal cell-derived factor 1 (SDF-1) using SDF-1 ELISA kit (cat. no. E-EL-R0922; Civic Bioscience Inc.) following the manufacturer's protocols.

Reverse transcription-quantitative polymerase chain reaction (RT-qPCR). Total RNA was extracted from BMMSCs using the RNeasy Mini Kit (Qiagen, Inc.), then the purity and concentration were determined spectrophotometrically. For each sample, $1 \mu \mathrm{g}$ of total RNA was reverse transcribed to cDNA using the PrimeScript ${ }^{\mathrm{TM}} \mathrm{RT}$ reagent kit (Takara Bio, Inc.). The normalized cDNA was then amplified with SYBR
Premix ExTM Taq II RT-PCR kit in Applied Biosystems 7,500 RT-PCR System. Thermocycling conditions were as follows: $95^{\circ} \mathrm{C}$ for $30 \mathrm{sec}$, followed by 40 cycles of $95^{\circ} \mathrm{C}$ for $5 \mathrm{sec}$ and $60^{\circ} \mathrm{C}$ for $30 \mathrm{sec}$. mRNA expression was calculated as previously reported (18), with GAPDH used as the housekeeping gene. The primer sequences were as follows: SDF-1 forward, 5'-GAGAGCCACATCGCCAGAGC-3' and reverse, 5'-GGA TCCACTTTAATTTCGGGTCAA-3'; runt-related transcription factor 2 (Runx2) forward, 5'-CACTGGCGCTGCAAC AAGA-3' and reverse, 5'-CATTCCGGAGCTCAGCAGAAT AA-3'; ALP forward, 5'-GCTCCCTTGTCTGGTCTTT-3' and reverse, 5'-GGACGCCGTGAAGCAGGTGA-3'; and GAPDH forward, 5'-AGCCGCATCTTCTTTTGCGTC-3' and reverse, 5'-TCATATTTGGCAGGTTTTTCT-3'.

Western blot analysis. Rat BMMSCs from cell culture were collected for western blot analysis. Proteins were extracted from the cells using Membrane protein isolation mammalian protein extraction reagent (Thermo Fisher Scientific, Inc.). Bicinchoninic acid assay was used to quantify protein concentration. A total of $25 \%$ volume of protein sample buffer was added, boiled for $5 \mathrm{~min}$ then proteins $(50 \mu \mathrm{g}$ loaded per lane) were separated via SDS-PAGE on an $8 \%$ gel. The separated proteins were then transferred to polyvinylidene difluoride membranes. Following blocking with $7 \%$ fat-free dry milk for $2.5 \mathrm{~h}$ at $4^{\circ} \mathrm{C}$, the membranes were incubated with primary antibodies against ALP (cat. no. 194297; 1:1,000; Abcam) and Runx2 (cat. no. 12556S; 1:1,000; Cell Signaling Technology, Inc.) at $4^{\circ} \mathrm{C}$ overnight. Following primary incubation, membranes were incubated with horseradish peroxidase-conjugated $\mathrm{IgG}$ secondary antibody (cat. no. 150077; 1:200; Abcam) for $1 \mathrm{~h}$. Membranes were then treated with enhanced chemiluminescent substrate (Thermo Fisher Scientific, Inc.) to visualize the bands. $\beta$-actin was used as an endogenous reference. Each experiment was performed three times.

Statistical analysis. The statistical analysis was performed by SPSS software (v.19.0; IBM Corp.) and the data were presented as mean \pm standard deviation. One-way analysis 
A

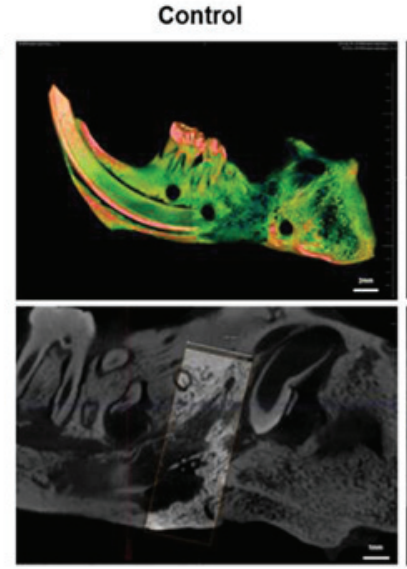

CGRP

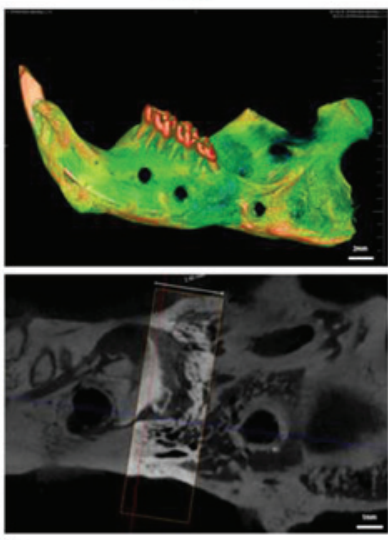

B

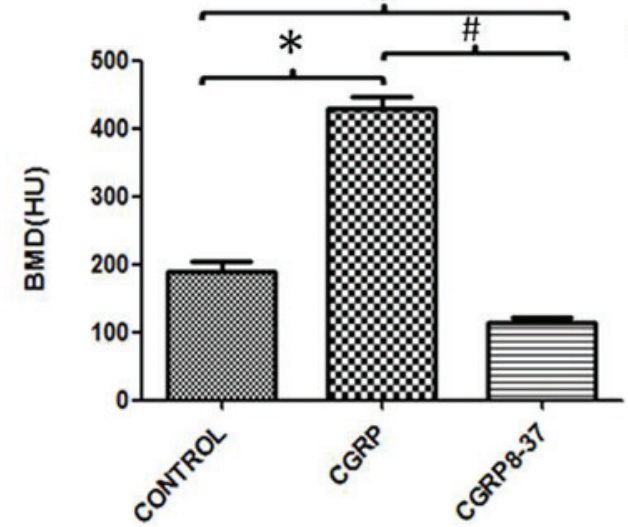

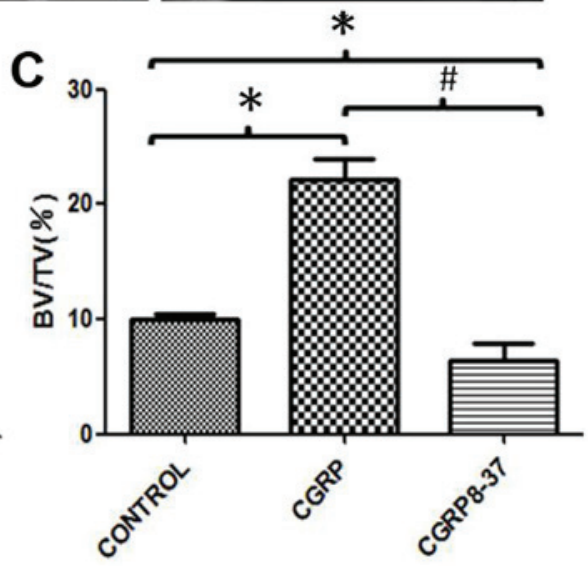

D Control CGRP

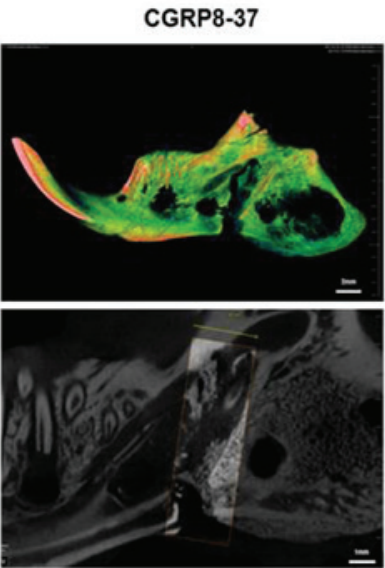

$*$
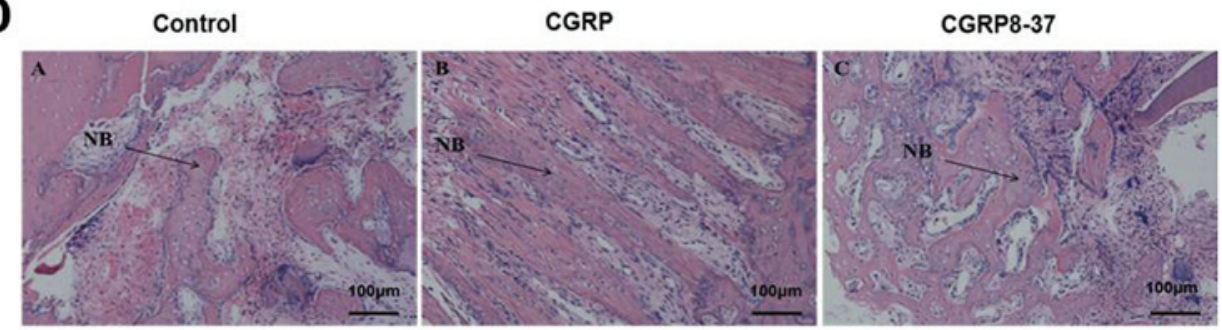

Figure 3. Effects of CGRP on bone regeneration in vivo. (A) Micro-computed tomography micrographs of rat mandibles. (B) Quantification of BMD and (C) BV/TV. (D) Histology analysis of each group. "P $<0.05$ vs. control; " $\mathrm{P}<0.05$ vs. CGRP. CGRP, calcitonin gene-related peptide; BMD, bone mineral density; $\mathrm{BV}$, bone volume; $\mathrm{TV}$, total volume.

of variance followed by the Neuman-Keuls post hoc test was used for analysis. $\mathrm{P}<0.05$ was considered to indicate statistical significance.

\section{Results}

Effects of CGRP on osteogenic differentiation and proliferation in vitro. Using a well-established method, rat BMMSCs were isolated. It was determined that morphology was typically fibroblastic at P0 and spindle-like at P3 (Fig. 1A). Immunofluorescence staining demonstrated that rat BMMSCs were positive for MSC markers CD90 and CD29 (Fig. 1A). In addition, rat BMMSCs differentiated into adipocytes using adipogenic medium or osteoblasts using osteogenic medium, as indicated by Oil Red or ARS, respectively (Fig. 1A and B). The osteoblast phenotype of the control, CGRP and CGRP8-37 treatment groups was confirmed by the presence of matrix mineralization.
After 21 days, osteogenic differentiation of BMMSCs was significantly enhanced by CGRP treatment and attenuated by CGRP8-37 (Fig. 1B and C). Runx2, SDF-1 and ALP mRNA expression levels were evaluated at 1, 3, 7, 14 and 21 days. mRNA expression began to rise from day 3 and peaked at day 14. At day 14, ALP expression in the CGRP group increased 4.2-fold ( $\mathrm{P}<0.05$; Fig. 1D), SDF-1 increased 3.8-fold $(\mathrm{P}<0.05$; Fig. $1 \mathrm{H})$ and Runx2 increased 3.2-fold $(\mathrm{P}<0.05$; Fig. 1E), compared to day 1. ALP and Runx2 expression of cells was further validated using western blot analysis (Fig. 1F and G). ELISA analysis further confirmed that concentrations of SDF-1 significantly increased in the CGRP group at day 7 and $14(\mathrm{P}<0.05$; Fig. 1I). The effect of CGRP on cell proliferation was assessed by the BrdU incorporation assay. CGRP significantly increased the percentage of $\mathrm{BrdU}^{+} \mathrm{BMMSCs}$ over the total number of cells, whilst CGRP8-37 significantly decreased the percentage of $\mathrm{BrdU}^{+}$ BMMSCs ( $\mathrm{P}<0.05$; Fig. 2). 
A
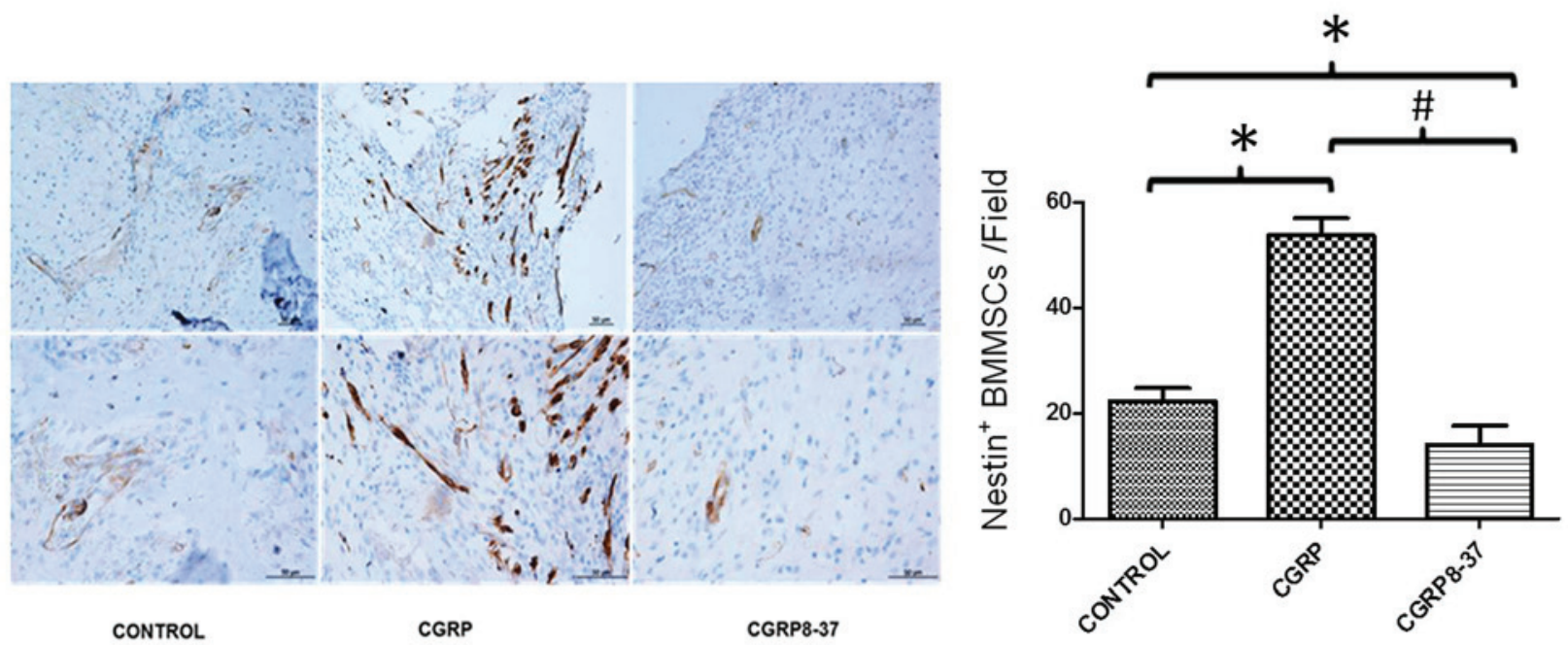

B

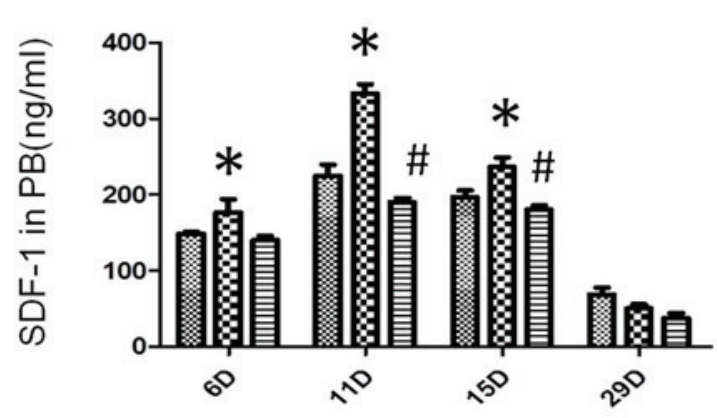

C

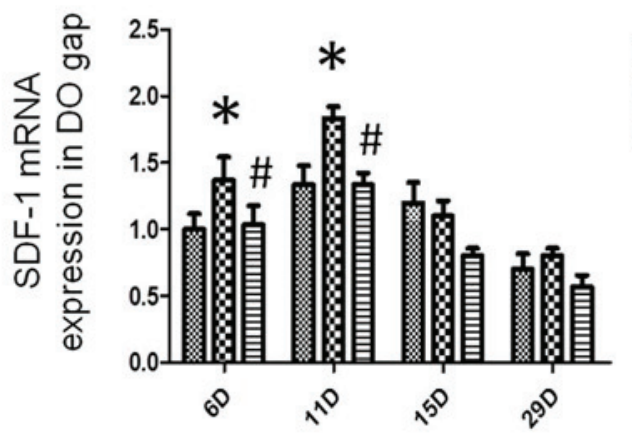

Figure 4. Effects of CGRP on bone regeneration in vivo. (A) Immunohistochemistry micrographs of Nestin ${ }^{+}$BMMSCs (brown) with quantification of Nestin ${ }^{+}$ BMMSCs in the DO region. (B) SDF-1 expression in PB and (C) DO region. ${ }^{*} \mathrm{P}<0.05$ vs. control; ${ }^{\#} \mathrm{P}<0.05$ vs. CGRP. CGRP, calcitonin gene-related peptide; BMMSCs, bone marrow mesenchymal stem cells; DO, distraction osteogenesis; PB, peripheral blood; SDF-1, stromal cell-derived factor 1; D, days.

Effects of CGRP on bone regeneration in vivo. All three groups demonstrated successful mandible lengthening. Micro-CT imaging determined that the distraction gaps were filled with newly formed bone in the control and CGRP groups, while only scattered bone trabeculae were present in the CGRP8-37 group (Fig. 3A). The BMD and BV/TV ratio in the CGRP group were significantly higher than other two groups, which indicated increased new bone generation $(\mathrm{P}<0.05$; Fig. 3B and $\mathrm{C})$. The morphology of new bone in the CGRP group was tighter and more continuous compared with the control group (Fig. 3D).

HE staining demonstrated that there was no inflammation in any group. The CGRP group had a large amount of woven bone in the distraction axial direction, which is associated with osteoblasts and new blood vessels. In addition, the trabeculae were orderly arranged. The CGRP8-37 group exhibited increased fibrous connective tissues and fewer bone masses and trabeculae. The trabeculae were disorderly arranged in the CGRP8-37 group (Fig. 3B).

To further investigate the relationship between new bone and Nestin ${ }^{+}$BMMSCs, immunohistochemistry was used to evaluate the migration of $\mathrm{Nestin}^{+} \mathrm{BMMSC}$ from the perivascular area to the DO gap. In the CGRP group, the Nestin ${ }^{+}$ BMMSCs were extensively distributed in bone generating areas of the callus whilst they were mainly distributed in the perivascular areas, rather than bone generating areas, in the other two groups (Fig. 4A). The number of Nestin ${ }^{+}$cells in bone generating areas were notably higher in the CGRP compared with the control $(\mathrm{P}<0.05$; Fig. $4 \mathrm{~A})$. In addition, SDF-1 expression in both the peripheral blood and regenerating region were significantly higher in the CGRP group compared with the other groups $(\mathrm{P}<0.05$; Fig. $4 \mathrm{~B}$ and $\mathrm{C})$. These findings suggested that CGRP treatment improved the migration of BMMSCs from the perivascular area to bone generating areas.

\section{Discussion}

The present study investigated the effects of CGRP throughout DO in vivo, as well as proliferation, differentiation and migration of BMMSCs in vitro. A modified titanium distractor device, similar to previously described devices $(19,20)$ was utilized to successfully establish the DO model. Micro-CT and histomorphometric analysis demonstrated that local injection of CGRP effectively increased and accelerated osteogenesis, whilst CGRP8-37 significantly impaired bone formation. In addition, Nestin ${ }^{+}$cells in the DO gap and SDF-1 expression was significantly increased following CGRP administration, which suggested that CGRP promoted the recruitment of BMMSCs to the new bone generating area. To support the in vivo study, in vitro results demonstrated that CGRP treatment modulated bone metabolism in osteoprogenitor differentiation and maturation. 
Neurotransmitters (e.g., substance P) have been proven to stimulate proliferation and osteogenic differentiation of MSCs in numerous animal models (29). Our previous study demonstrated that local injection of substance $\mathrm{P}$ accelerates the generation of bone during mandibular distraction in rats (21). Hong et al (8) demonstrated that systemic administration of substance $\mathrm{P}$ promotes the mobilization of BMMSC to the bone generating regions. Therefore, the present study hypothesized that CGRP may have an effect on sensory nerves serving a similar role in promoting the mobilization of MSCs akin to substance P.

CGRP belongs to the calcitonin superfamily of peptides consisting of calcitonin, amylin, adrenomedullin, adrenomedullin 2 (intermedin) and calcitonin-receptor-stimulating peptide. Calcitonin is mainly produced by thyroid $\mathrm{C}$ cells whilst CGRP is secreted and stored in the nervous system. Calcitonin can inhibit osteoclast motility and cause bone resorption disorders; however, there is limited evidence of the direct effect of calcitonin on osteoblasts. Notably, neuropeptides, such as CGRPs, are pivotal in suppressing bone resorption via the receptor activator of nuclear factor $-\kappa B /$ osteoprotegerin pathway (22). CGRPs are less potent than calcitonin in inducing hypocalcemia by several orders of magnitude $(23,24)$ but it has been suggested that CGRP stimulates osteoblasts and bone formation. Activation of osteoblastic CGRP receptors enhances the proliferation of osteoblasts in vitro (25) and treatment of the osteocalcin promoter on transgenic mice resulted in an enhanced bone mass level (26). It has also been reported that exogenous CGRP can accelerate the proliferation of MSCs in the logarithmic growth phase (27). Furthermore, CGRP modulates the differentiation of SCs towards osteoblasts in vivo (28) and also regulates cell differentiation in the induced pluripotent and embryoid body stages (29). Therefore, CGRP has an important role in mediating osteoblasts in cellular and autocrine activities. The CGRP-containing fibers distribute abundantly in bone marrow and periosteum, which suggests a mechanistic connection between osteoblasts and CGRP-containing nerve fibers. In addition, CGRP-containing fibers in the epiphyseal trabeculae are rarely covered by the Schwann cell sheaths which may promote the interaction of BMMSCs with CGRP released from the fibers (29). Another characteristic of CGRP-containing fibers is their persistent contact with the bone surface. The links between CGRP-containing fibers and osteoblasts by the uncovered varicosities are maintained by bone generation. After bone injury or fracture, the CGRP-containing fibers may trigger bone regeneration around the blood vessels. However, CGRP mRNA and protein expression in osteoblasts has also been observed (28). Therefore, it is highly reasonable that CGRP may influence osteoblastogenesis and the metabolism of bone formation by regulating BMMSC activity. Our future work will determine other osteogenic markers and further investigate the molecular mechanisms underlying the role of CGRP in osteogenic differentiation and BMMSC recruitment.

In conclusion, the present study determined that CGRP could accelerate bone formation during DO. These findings may provide an insight into the mechanisms underlying bone regeneration promoted by sensory nerves, and also provides evidence for the efficiency and safety of DO treatment.

\section{Acknowledgements}

Not applicable.

\section{Funding}

This study was supported by the National Natural Science Foundation of China (grant nos. 81771046 and 81270015), Shanghai Talent Development (grant no. 2018042) and Shanghai Summit and Plateau Disciplines. The funders had no role in study design, data collection and analysis, or preparation of the manuscript.

\section{Availability of data and materials}

The datasets generated and/or analysed during the current study are available from the corresponding author on reasonable request.

\section{Authors' contributions}

LW and WY designed and supervised the project. SJ, SZ, $\mathrm{XW}, \mathrm{ZY}$ and YS performed the experiments. AG, RH, DL and $\mathrm{KH}$ analyzed the data. SJ, XW, AG and LW wrote the article. All authors read and approved the final manuscript.

\section{Ethics approval and consent to participate}

The protocol for animal experimentation was approved by the Institutional Ethics Committee at the Fourth Military Medical University (approval no. ky-034).

\section{Patient consent for publication}

Not applicable.

\section{Competing interests}

The authors declare that they have no competing interests.

\section{References}

1. van Strijen PJ, Breuning KH, Becking AG, Perdijk FB and Tuinzing DB: Complications in bilateral mandibular distraction osteogenesis using internal devices. Oral Surg Oral Med Oral Pathol Oral Radiol Endod 96: 392-397, 2003.

2. Garcia-Castellano JM, Diaz-Herrera P and Morcuende JA: Is bone a target-tissue for the nervous system? New advances on the understanding of their interactions. Iowa Orthop J 20: 49-58, 2000.

3. Suzuki A, Uemura T and Nakamura H: Control of bone remodeling by nervous system. Neural involvement in fracture healing and bone regeneration. Clin Calcium 20: 1820-1827, 2010 (In Japanese).

4. Anderson JJ, Woelffer KE, Holtzman JJ and Jacobs AM: Bisphosphonates for the treatment of Charcot neuroarthropathy J Foot Ankle Surg 43: 285-289, 2004.

5. Du ZJ, Wang L, Lei DL, Liu BL, Cao J, Zhang P and Ma Q: Nerve growth factor injected systemically improves the recovery of the inferior alveolar nerve in a rabbit model of mandibular distraction osteogenesis. Br J Oral Maxillofac Surg 49: 557-561, 2011.

6. Wang L, Cao J, Lei DL, Cheng XB, Zhou HZ, Hou R, Zhao YH and Cui FZ: Application of nerve growth factor by gel increases formation of bone in mandibular distraction osteogenesis in rabbits. Br J Oral Maxillofac Surg 48: 515-519, 2010. 
7. Wang L, Shi X, Zhao R, Halloran BP, Clark DJ, Jacobs CR and Kingery WS: Calcitonin-gene-related peptide stimulates stromal cell osteogenic differentiation and inhibits RANKL induced NF-kappaB activation, osteoclastogenesis and bone resorption. Bone 46: 1369-1379, 2010.

8. Hong HS, Lee J, Lee E, Kwon YS, Lee E, Ahn W, Jiang MH, Kim JC and Son Y: A new role of substance $\mathrm{P}$ as an injury-inducible messenger for mobilization of CD29(+) stromal-like cells. Nat Med 15: 425-435, 2009.

9. Yu X, Lv L, Zhang J, Zhang T, Xiao C and Li S: Expression of neuropeptides and bone remodeling-related factors during periodontal tissue regeneration in denervated rats. J Mol Histol 46: 195-203, 2015.

10. Sample SJ, Heaton CM, Behan M, Bleedorn JA, Racette MA, Hao Z and Muir P: Role of calcitonin gene-related peptide in functional adaptation of the skeleton. PLoS One 9: e113959, 2014

11. Wimalawansa SJ: Calcitonin gene-related peptide, and its receptors: Molecular genetics, physiology, pathophysiology and therapeutic potentials. Endocr Rev 17: 533-585, 1996.

12. Yang Z, Wu B, Jia S, Zhao Y, Hou R, Liu X, Wang X, Chen L, Yang X, Lei D and Wang L: The mechanically activated p38/MMP-2 signaling pathway promotes bone marrow mesenchymal stem cell migration in rats. Arch Oral Biol 76: 55-60, 2017.

13. Yang ZH, Wu BL, Ye C, Jia S, Yang XJ, Hou R, Lei DL and Wang L: Targeting P38 pathway regulates bony formation via MSC recruitment during mandibular distraction osteogenesis in rats. Int J Med Sci 13: 783-789, 2016.

14. Wang L, Zhao Y, Liu Y, Akiyama K, Chen C, Qu C, Jin Y and Shi S: IFN- $\gamma$ and TNF- $\alpha$ synergistically induce mesenchymal stem cell impairment and tumorigenesis via NFKB signaling. Stem Cells 31: 1383-1395, 2013.

15. Wang L, Liu S, Zhao Y, Liu D, Liu Y, Chen C, Karray S, Shi S and Jin Y: Osteoblast-induced osteoclast apoptosis by fas ligand/FAS pathway is required for maintenance of bone mass. Cell Death Differ 22: 1654-1664, 2015.

16. Wu B, Wang L, Yang X, Mao M, Ye C, Liu P, Yang Z, Yang X, Lei D and Zhang C: Norepinephrine inhibits mesenchymal stem cell chemotaxis migration by increasing stromal cell-derived factor-1 secretion by vascular endothelial cells via NE/abrd3/JNK pathway. Exp Cell Res 349: 214-220, 2016.

17. Villa I,Melzi R,Pagani F, Ravasi F, Rubinacci A and Guidobono F: Effects of calcitonin gene-related peptide and amylin on human osteoblast-like cells proliferation. Eur J Pharmacol 409: 273-278, 2000 .

18. Livak KJ and Schmittgen TD: Analysis of relative gene expression data using real-time quantitative PCR and the 2(-Delta Delta C(T)) method. Methods 25: 402-408, 2001.

19. Wang T, Cao J, Du ZJ, Zhang YB, Liu YP, Wang L and Lei DL: Effects of sympathetic innervation loss on mandibular distraction osteogenesis. J Craniofac Surg 23: 1524-1528, 2012.
20. Zhang YB, Wang L, Jia S, Du ZJ, Zhao YH, Liu YP and Lei DL: Local injection of substance $P$ increases bony formation during mandibular distraction osteogenesis in rats. Br J Oral Maxillofac Surg 52: 697-702, 2014

21. Imai S, Tokunaga $Y$, Maeda T, Kikkawa M and Hukuda $S$ : Calcitonin gene-related peptide, substance $\mathrm{P}$ and tyrosine hydroxylase-immunoreactive innervation of rat bone marrows: An immunohistochemical and ultrastructural investigation on possible efferent and afferent mechanisms. J Orthop Res 15: 133-140, 1997.

22. Yoo YM, Kwag JH, Kim KH and Kim CH: Effects of neuropeptides and mechanical loading on bone cell resorption in vitro. Int J Mol Sci 15: 5874-5883, 2014.

23. MacIntyre I: Amylinamide, bone conservation and pancreatic beta cells. Lancet 2: 1026-1027, 1989.

24. Tippins JR, Morris HR, Panico M, Etienne T, Bevis P, Girgis S, MacIntyre I, Azria M and Attinger M: The myotropic and plasma-calcium modulating effects of calcitonin gene-related peptide (CGRP). Neuropeptides 4: 425-434, 1984.

25. Cornish J, Callon KE, Lin CQ, Xiao CL, Gamble GD, Cooper GJ and Reid IR: Comparison of the effects of calcitonin gene-related peptide and amylin on osteoblasts. J Bone Miner Res 14: $1302-1309,1999$

26. Ballica R, Valentijn K, Khachatryan A, Guerder S, Kapadia S, Gundberg C, Gilligan J, Flavell RA and Vignery A: Targeted expression of calcitonin gene-related peptide to osteoblasts increases bone density in mice. J Bone Miner Res 14: 1067-1074, 1999

27. Xu G and Jiang D: The role and mechanism of exogenous calcitonin gene-related peptide on mesenchymal stem cell proliferation and osteogenetic formation. Cell Biochem Biophys 69: 369-378, 2014.

28. Fang Z, Yang Q, Xiong W, Li GH, Liao H, Xiao J and Li F: Effect of CGRP-adenoviral vector transduction on the osteoblastic differentiation of rat adipose-derived stem cells. PLoS One 8: e72738, 2013

29. Nagao S, Goto T, Kataoka S, Toyono T, Joujima T, Egusa H, Yatani H, Kobayashi S and Maki K: Expression of neuropeptide receptor mRNA during osteoblastic differentiation of mouse iPS cells. Neuropeptides 48: 399-406, 2014.

This work is licensed under a Creative Commons Attribution-NonCommercial-NoDerivatives 4.0 International (CC BY-NC-ND 4.0) License. 